\title{
2 Theoretische Grundlagen: Tempus, Aspekt und Aktionsart
}

Alle menschlichen Kulturen haben ein Verständnis von Zeit (Comrie 1985: 3-4).

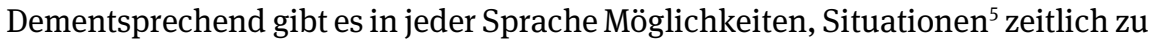
verorten (ebd.: 7). Grundlegend ist dabei die Opposition zwischen Vergangenheit und Nicht-Vergangenheit (ebd.: 2-4). Nicht in allen Sprachen existiert eine grammatische Kategorie zur zeitlichen Einordnung von Situationen (ebd.: 4). In diesen Sprachen verorten stattdessen lexikalische Zeichen (wie beispielsweise Adverbien) Situationen in der Zeit (ebd.: 8). Demgegenüber findet man in vielen Sprachen der Welt eine grammatische Markierung zur zeitlichen Verortung von Situationen (ebd.). Zwei wesentliche grammatische Kategorien zur zeitlichen Verortung von Situationen sind Tempus und Aspekt. Inwieweit diese Begriffe zusammenhängen, wird in der Forschungsliteratur zum Teil kontrovers diskutiert. Deutlich wird dies vor allem im Umgang mit dem Perfekt im Deutschen, das zum Teil als reines Tempus oder reiner Aspekt beschrieben wird und zum Teil als kategoriale Mischform, die sowohl temporale als auch aspektuelle Eigenschaften in sich vereint (vgl. dazu Heinold 2015: 102). Eisenberg argumentiert sogar dafür, dass Tempus grundsätzlich nicht nur rein deiktisch-zeitlich aufzufassen ist, sondern auch „ein aspektuelles Verhältnis signalisiert“ (1994: 123). Eine Definition von Tempus und Aspekt muss also immer auch eine Abgrenzung dieser Begriffe zueinander beinhalten (vgl. dazu auch Breuer/Dorow 1996: 13).

Tempus lässt sich vorrangig durch dessen deiktisch-zeitliche Funktion (Comrie 1985: 14 und Binnick 2001: 557) vom Aspekt unterscheiden. Deiktisch-zeitlich meint hierbei die Verweisfunktion des Tempus von einer Zeitstufe auf eine andere. Das Tempus hat also eine situationsexterne Perspektive, da es - vereinfacht gesagt - von der Äußerungszeit auf die Situationszeit verweist. Davon abzugrenzen ist die situationsinterne (d.h. nicht-deiktische) Perspektive des Aspekts (Breuer/Dorow 1996: 17). ${ }^{6}$

5 Ich verwende den Begriff Situation wie Lindstedt (2001: 768) und Fischer (2018: 166) als Oberbegriff für „anything that a sentence denotes“ (Lindstedt 2001: 768), d. h. für Ereignisse, Prozesse, Zustände etc.

6 Auf die häufig verwendeten Begriffe der situationsexternen Perspektive des Tempus beziehungsweise der situationsinternen Perspektive des Aspekts wird in dieser Arbeit jedoch verzichtet, um begriffliche Verwirrungen mit der Einteilung des Aspekts in perfektiv und imperfektiv zu vermeiden, die sich durch genau diese vom Sprecher eingenommene externe beziehungsweise interne Perspektive unterscheiden. 
Im Gegensatz zum Tempus verweist der Aspekt nicht von einer Zeitstufe auf eine andere, sondern perspektiviert die innere zeitliche Charakteristik eines Ereignisses. Er drückt also aus, ob ein Ereignis als abgeschlossen (=perfektiv) oder unabgeschlossen (=imperfektiv) betrachtet wird, d. h. im Verlauf befindend oder nicht im Verlauf befindend. Gerade die aspektuelle Kategorie abgeschlossen (=perfektiv) und die temporale Kategorie Vergangenheit werden in der Literatur nicht einheitlich gebraucht und häufig nicht klar voneinander getrennt. Der Grund für diese begriffliche Verwirrung liegt in der Verzahnung der beiden Kategorien. Damit eine Situation als abgeschlossen beurteilt werden kann, muss sie ,von außen' betrachtet werden, d. h. zeitlich nach der Situation und nicht während sie noch Gültigkeit hat. Obwohl der Verweis auf frühere Zeitstufen und Abgeschlossenheit häufig korrelieren, sind Aspekt und Tempus als konzeptuell eigenständig zu sehen. Auf diese Verbindung aus Aspekt und Tempus gehe ich in den Kapiteln 2.2.2 und 2.2.3 noch näher ein.

Allerdings ist nicht nur die Differenzierung zwischen Aspekt und Tempus prekär, auch die Verwendung des Begriffs Aspekt selbst sorgt in der Literatur häufig für Unklarheit. Zum einen liegt dies an der nicht immer eindeutigen Abgrenzung zum Terminus Aktionsart, zum anderen an der unterschiedlichen Auffassung, ob Aspekt ein einzelsprachliches Phänomen ist, das überwiegend durch Flexion ausgedrückt wird und vorrangig in slawischen Sprachen auftritt (Admoni 1982: 172), oder ein übereinzelsprachliches, das je nach Sprache viele verschiedene systematische morphologische und lexikalische Realisierungsmöglichkeiten kennt (vgl. hierzu u. a. Lyons 1971, Comrie 1981 und Bybee 1985).

Aufgrund der häufig zu beobachtenden Vermengung von Aktionsart und Aspekt erscheint es besonders wichtig, diese Begriffe klar voneinander zu trennen. Obwohl beide einen ähnlichen Beitrag zur Satzbedeutung liefern, sind sie nicht identisch. Als grundsätzliche Unterscheidung lässt sich feststellen, dass die Aktionsart der lexikalischen Semantik des Verbs bereits inhärent ist, während der Aspekt formal am Verb realisiert wird und paradigmatisch bei konstantem Verbstamm variiert. Die polnischen Beispielsätze (4) sollen dies verdeutlichen.

(4) a. Jan przeczytał książkę.

Jan PERF las ein Buch.

b. Jan czytał książkę.

Jan IMPERF las ein Buch. ${ }^{7}$

7 Ich danke Natalia Boltz für die polnische Übersetzung der Beispielsätze. 
Die Aktionsart ist in beiden Sätzen identisch (=Verb ändert sich nicht). Der Aspekt ist jedoch in Beispiel (4a) perfektiv (ausgedrückt durch das Präfix prze-), in Beispiel (4b) imperfektiv.

Ob Aspekt durch Präfigierung markiert werden muss wie in den klassischen Aspektsprachen oder eine übereinzelsprachliche Kategorie ist, die durch verschiedene morphologische oder lexikalische Mittel ausgedrückt werden kann, wird noch zu zeigen sein. Neben der inhaltlichen Nähe der beiden Begriffe ist auch die begriffsgeschichtlich enge Verwandtschaft der beiden Termini ausschlaggebend für deren unklare Verwendung. Obwohl sich die Slawistik bereits seit dem 19. Jahrhundert mit Aspekt befasst, grenzte erst Agrell (1908) Aspekt von Aktionsart ab. Doch auch im Anschluss daran stellte sich keine einheitliche und differenzierende Verwendung der beiden Begriffe ein. In der deutschen Literatur wurden die beiden von Aktionsart und Aspekt vermittelten Bereiche teilweise gemeinsam unter dem Begriff der Aktionsart zusammengefasst. Pollak bezeichnet etwa die Aktionsart als lexikalische Aktionsart, den Aspekt dagegen als grammatische Aktionsart (1967: 414-417). Sasses Vorschlag, diese Ambiguität durch die einheitliche Verwendung des aus dem anglo-sächsischen Raum stammenden Begriffs aspect aufzulösen (Sasse 1991: 31-32), ist nicht zielführend. Dabei finden sich die Begriffe lexical aspect für die Aktionsart und grammatical aspect für den eigentlichen Aspekt (vgl. u. a. Li/Shirai 2000: 3). So liegt ein weiterer Grund für die konfuse Verwendung der Begriffe in genau dieser Gleichnennung von Aktionsart und Aspekt als aspect. Um eine begriffliche Verwirrung zu vermeiden, werde ich in dieser Arbeit die sprecherunabhängige (d. h. objektive) Perspektive, die in der Verbbedeutung selbst liegt, als Aktionsart und die sprecherabhängige (d. h. subjektive) Perspektive, die durch etwas Externes zur eigentlichen Verbbedeutung vermittelt wird, als Aspekt bezeichnen.

In Kapitel 2.2.1 werde ich dafür argumentieren, Aspekt als übereinzelsprachliches Phänomen zu begreifen, das in vielen Sprachen Ausdruck findet. Hierzu wird auch der Standpunkt Eisenbergs empirisch überprüft, Tempus und Aspekt seien zumindest im Deutschen in den meisten Fällen miteinander verwoben. Denkbar wäre, dass im Alemannischen durch Tempus-Variationen unterschiedliche Aspekte ausgedrückt werden. Für eine Darstellung der theoretischen Grundlagen soll es aber zunächst ausreichen, Tempus als rein deiktisch-zeitlich zu begreifen und von den nicht-deiktisch-zeitlichen Kategorien Aktionsart und Aspekt abzugrenzen. Nach einer Definition von Tempus werde ich in diesem Kapitel Aspekt definieren und daraufhin die in dieser Arbeit unterschiedenen Aspekte vorstellen. Im Anschluss daran liefere ich eine Definition von Aktionsart, indem ich diese vom Aspekt abgrenze, und stelle danach die für diese Arbeit relevanten Aktionsarten vor. 


\subsection{Tempus}

\subsubsection{Das Reichenbach'sche Tempussystem}

Tempus wird von den meisten Grammatiken im Deutschen als obligatorisch angesehen, wobei es entweder analytisch (durch Hilfsverben) oder synthetisch (in Form von Flexion) gebildet wird. ${ }^{8}$ Seine Hauptfunktion ist ein zeitlicher Verweis von einer Zeitstufe auf eine andere. Die deiktische Funktion des Tempus lässt sich besonders gut durch das von Reichenbach aufgestellte System verdeutlichen (1947). Er unterscheidet drei Zeitpunkte, die auf einer von der Vergangenheit über die Gegenwart in die Zukunft laufenden Zeitachse liegen: den Sprech[zeit]punkt, den Ereignis[zeit]punkt und den Referenz[zeit]punkt (1947: 273). Häufig finden sich in der Literatur anstelle von Zeitpunkten auch Begrifflichkeiten wie Zeitspannen (vgl. hierzu u. a. Ehrich/Vater 1989: 119). Dies wird dadurch begründet, dass grundsätzlich jede beschriebene Situation, egal ob punktuell oder durativ, eine zeitliche Ausdehnung besitzt. Ich möchte in dieser Arbeit untersuchen, inwieweit Tempus und Aspekt im Alemannischen gekoppelt sind, d. h. ob ein Tempus neben der Verweisfunktion auch ausdrücken kann, ob eine Situation als abgeschlossen, also ganzheitlich, oder als unabgeschlossen, also nicht-ganzheitlich, betrachtet wird. Um diesen verschiedenen Perspektivierungen von Situationen die passenden Termini Zeitpunkt und Zeitspanne zuordnen zu können und eine begriffliche Verwirrung zu vermeiden, bezeichne ich die in dieser Hinsicht undifferenzierten Reichenbach'schen Zeitpunkte als Sprechzeit, Ereigniszeit und Referenzzeit.

Unter Sprechzeit verstehe ich mit Reichenbach die Zeit, zu der eine Äußerung getätigt (beziehungsweise ein Satz geschrieben) wird (Reichenbach 1947: 273). Diese ist nicht mit der Gegenwart gleichzusetzen, auch wenn dies die intuitive Auffassung der Sprechzeit ist. Breuer/Dorow (1996) verweisen darauf, dass die Sprechzeit die tatsächliche Zeitdauer der Äußerung oder Satzverwendung meint. Gegenwart dagegen ist die subjektive Wahrnehmung des Augenblicks. Diese wird in psychologischen Untersuchungen zwischen Sekundenbruchteilen und

8 Eine Ausnahme stellt hier vor allen Dingen die Dissertation von Mugler (1988) dar. Darin spricht Mugler dem Präsens den Status eines Tempus ab, da es sich auf Vergangenheit, Gegenwart und Zukunft beziehen kann und sich formal durch das Fehlen einer Flexionsendung zum Ausdruck von Tempus auszeichnet (1988: 158). Demzufolge wäre Tempus keine obligatorische Kategorie, die in jeder Verbform vermittelt wird, sondern fakultativ. Thieroff zeigt allerdings, dass Präsens keine merkmallose Form ist und auch nicht grundsätzlich als Ausdruck von Vergangenheit dienen kann (1992: 64). 
circa drei Sekunden Länge angegeben. Die Sprechzeit kann allerdings länger oder kürzer sein als der Moment der Gegenwart (Breuer/Dorow 1996: 7).

Als Ereigniszeit betrachtet Reichenbach den Zeitpunkt, zu dem das beschriebene Ereignis (also die Verbalhandlung) tatsächlich stattfindet (Reichenbach 1947: 273). Die Referenzzeit wiederum beschreibt einen Punkt, der relativ zu den beiden anderen Zeiten gesetzt werden kann (ebd.). Er kann also vor, nach oder zwischen den anderen beiden Zeiten liegen oder mit einer der beiden zusammenfallen. Während die Ereigniszeit also den objektiven Zeitpunkt eines Ereignisses angibt, verweist die Referenzzeit auf einen vom Sprecher subjektiv festgelegten, d. h. frei wählbaren, Zeitpunkt, von dem die Ereigniszeit aus betrachtet wird. Während es bei Tempora wie Präsens und Präteritum auf den ersten Blick nicht notwendig erscheint, eine Referenzzeit anzunehmen, wird deren Wichtigkeit für die Beschreibung von Tempora beim Plusquamperfekt ersichtlich.

(5) Melanie kam um zwei Uhr bei Max vorbei, aber Max war um zwei Uhr bereits gegangen.

Der Bedeutungsunterschied der beiden Teilsätze in (5) lässt sich mit der Distinktion Sprechzeit/Ereigniszeit alleine nicht erklären. Die Ereigniszeit beider beschriebener Ereignisse liegt (vereinfacht gesagt) in der Vergangenheit, die Sprechzeit in der Gegenwart. Erst durch das Hinzuziehen eines weiteren Zeitpunktes kann ein Bedeutungsunterschied festgemacht werden. So gibt die Nominalgruppe um zwei Uhr in beiden Sätzen nicht die Ereigniszeit an, sondern die Referenzzeit. Während diese im ersten Teilsatz zeitgleich zur Ereigniszeit ist, liegt sie im zweiten Teilsatz zeitlich nach der Ereigniszeit. In dem einen Fall kommt Melanie also um zwei Uhr vorbei, im anderen ist Max zu irgendeiner Zeit vor zwei Uhr gegangen. Demzufolge ergibt sich für den ersten Teilsatz folgende Anordnung der Zeiten: Ereigniszeit=Referenzzeit ('um zwei Uhr') > Sprechzeit ('zeitlich nach zwei Uhr'); für den zweiten Teilsatz folgende Anordnung: Ereigniszeit ('zeitlich vor zwei Uhr') > Referenzzeit ('um zwei Uhr') > Sprechzeit ('zeitlich nach zwei Uhr').

Dass die Referenzzeit auch bei den Tempora Präsens, Präteritum, Perfekt und Futur I anzunehmen ist, zeigen unter anderem Ehrich/Vater (1989). Mit Hilfe der Referenzzeit erklären sie die Variation von Perfekt und Präteritum. Demnach fallen beim Präteritum Referenz- und Ereigniszeit zusammen, die beide zeitlich vor der Sprechzeit liegen, und beim Perfekt Referenz- und Sprechzeit, die zeitlich nach der Ereigniszeit lokalisiert sind (Ehrich/Vater 1989: 124). Dieser Ansatz erlaubt es, den Gegenwartsbezug des Perfekts theoretisch zu erfassen. Fischer (2018) schreibt unter anderem auf Ehrich/Vater (1989) Bezug nehmend dem 
Perfekt den Ausdruck des retrospektiven Aspekts zu (2018: 197). Demnach entstehe der retrospektive Aspekt, indem die Referenzzeit der Ereigniszeit nachgeordnet und zeitgleich zum Folgezustand einer Situation lokalisiert wird. Der Nachzustand diene als Bezugspunkt für die Referenzzeit, von der aus die Verbalsituation retrospektiv betrachtet werden kann (ebd.). Ausgehend von dieser theoretischen Grundlage möchte ich in Kapitel 3.2.2 überprüfen, ob das Perfekt auch im Badischalemannischen einen Gegenwartsbezug hat.

\subsubsection{Absolute, relative und absolut-relative Tempora}

Unklar ist also, ob für die Beschreibung der Funktion des Perfekts ein Referenzpunkt angenommen werden muss. Für eine Beschreibung des Plusquamperfekts ist dieser jedoch notwendig, um dessen zweifachen Verweis erfassen zu können. Dabei spielt bei Tempora wie Plusquamperfekt und Futur II in der Literatur häufig die Unterscheidung in absolute, relative und absolut-relative Tempora eine Rolle (vgl. hierzu u. a. Breuer/Dorow 1996 und Comrie 1985) - also Tempora, die sich einer einfachen Zuordnung als Ausdruck von Vergangenheit, Gegenwart oder Zukunft entziehen.

Ich betrachte nach Comrie (1985) als absolute Tempora die Tempora, die die Gegenwart (vereinfacht gesagt also die Sprechzeit) als deiktisches Zentrum haben und von dieser in die Vergangenheit, die Gegenwart oder die Zukunft verweisen (Comrie 1985: 36). Dabei ist irrelevant, ob von der Sprechzeit direkt auf die in der Vergangenheit, Gegenwart oder Zukunft liegende Ereigniszeit verwiesen wird oder auf eine zeitgleich zur Sprechzeit lokalisierte Referenzzeit, die wiederum in die Vergangenheit, Gegenwart oder Zukunft verweist. Bei absoluten Tempora ist die Referenzzeit also immer mit Ereignis- oder Sprechzeit identisch. ${ }^{9}$ Aus diesem Grund betrachte ich die Tempora Präsens, Präteritum, Perfekt und Futur I des Standarddeutschen als absolute Tempora, unabhängig davon, ob die Referenzzeit zeitgleich zur Ereignis- oder zur Sprechzeit zu situieren ist. Absolute Tempora können demnach ohne weiteren Kontext geäußert oder geschrieben werden, da die Sprechzeit ein unabhängiges deiktisches Zentrum bildet.

Als relative Tempora betrachte ich Tempora, deren deiktisches Zentrum erst durch den Kontext festgelegt wird. Genau wie die absoluten Tempora verweisen auch die relativen von ihrem deiktischen Zentrum in die Vergangenheit, die Gegenwart oder die Zukunft (Comrie 1985: 56). In den europäischen Sprachen gibt es keine relativen Tempora. Allerdings gibt es Verbformen, deren Bezugspunkt

9 Beim Präsens als absolutes Tempus fallen alle drei Reichenbach'schen Zeiten zusammen. 
kontextuell vorgegeben ist. Im Deutschen und Englischen sind dies infinite Verbformen (Breuer/Dorow 1996: 20). Infinitiv und Partizip I drücken Gleichzeitigkeit aus, das Partizip II Vorzeitigkeit. Je nach Kontext kann dieselbe infinite Verbform also in Vergangenheit, Gegenwart oder Zukunft (ausgehend von der Gegenwart) verweisen (ebd.).

Das Partizip I der Sätze (6) drückt Gleichzeitigkeit aus. Abhängig vom jeweiligen finiten Verb ist dies die Vergangenheit (6a), die Gegenwart (6b) oder die Zukunft (6c).

(6) a. Auf den Zug wartend trank das Mädchen eine heiße Schokolade.

b. Auf den Zug wartend trinkt das Mädchen eine heiße Schokolade.

c. Auf den Zug wartend wird das Mädchen eine heiße Schokolade trinken.

Die Ereigniszeit ändert sich bei relativen Tempora also von Kontext zu Kontext (hier durch die Änderung des finiten Verbs); bei absoluten Tempora bleibt sie identisch.

Absolut-relative Tempora bilden, wie aus deren Bezeichnung bereits hervorgeht, eine Mischform zur Distinktion absolut/relativ. Ich betrachte Tempora als absolut-relativ, wenn sie neben der Sprechzeit als deiktisches Zentrum ein zusätzliches vom Kontext festgelegtes Zentrum besitzen (Comrie 1985: 65). Diese Tempora verorten ein Ereignis also nicht nur relativ (d. h. vorher oder nachher) zur Sprechzeit, sondern zudem relativ (d. h. vorher oder nachher) zur vom Kontext gegebenen Referenzzeit, die bei diesen Tempora von Ereignis- und Sprechzeit verschieden ist. Für das Plusquamperfekt ergibt sich nach Comrie somit die Bedeutung „past in the past“ - es wird also ein Ereignis ausgedrückt, das zeitlich vor der Sprechzeit liegt und vor einer wiederum selbst vor der Sprechzeit gesetzten Referenzzeit (Comrie 1985: 65). Durch absolut-relative Tempora lassen sich komplexe zeitliche Verhältnisse ausdrücken, die über die Beschreibung von Vergangenem, Gegenwärtigem und Zukünftigem hinausgehen. Durch deren zweites deiktisches Zentrum (die Referenzzeit) lassen sich die Ereignisse nicht nur in Vergangenheit, Gegenwart oder Zukunft situieren, sondern auch chronologisch zu anderen Ereignissen. Comrie führt als Beispielsatz „John arrived; Mary left“ (Comrie 1985: 67) an und verweist hierbei auf die Indeterminiertheit in Bezug auf die zeitliche Anordnung der beiden Teilsätze zueinander. Hierbei ist unklar, ob Mary vor, nach oder während Johns Ankunft ging. Demgegenüber erlaubt der Satz „John arrived; Mary had left“ (Comrie 1985: 67) eine eindeutige Interpretation, wobei Mary gegangen ist, bevor John ankam.

Ein Ziel dieser Arbeit ist es zu zeigen, dass es sich bei Präteritum und Perfekt um absolute und bei Plusquamperfekt und Doppelperfekt um absolut-relative 
Tempora handelt. Präteritum und Perfekt wären demzufolge Tempora der einfachen Vergangenheit, Doppelperfekt und Plusquamperfekt Tempora der Vorvergangenheit. Ich versuche zu zeigen, dass auch viele vermeintlich absolute Verwendungen von Plusquamperfekt und Doppelperfekt, bei denen keine explizit in der Vergangenheit liegende Referenzzeit zu erkennen ist, eine Vorvergangenheitsbedeutung besitzen (Kap. 4.2.2). Bezogen auf das obige Beispiel könnte man als absolute Verwendung eines Plusquamperfekts die singuläre Äußerung „Mary had left“ anführen, bei der erst durch Hinzuziehen des Kontextes eine mögliche zusätzliche Referenzzeit aufgedeckt werden kann.

\subsubsection{Die Funktion von Vorvergangenheitstempora}

Ich möchte nun auf die Frage eingehen, warum der sprachliche Ausdruck einer zeitlichen Vorvergangenheit überhaupt notwendig ist. Die meisten Arbeiten zum Thema Tempus übergehen diese Frage und setzen die Notwendigkeit einer Vorvergangenheit einfach voraus. Dies ist auf den ersten Blick alles andere als selbstverständlich. Schließlich ist bei der Aneinanderreihung mehrerer chronologisch aufeinander folgender Ereignisse (sei es bei einer Erzählung, einem Bericht oder dergleichen) jedes Ereignis vor beziehungsweise nach einem anderen. Doch nicht jedes dieser Ereignisse wird mit einem Tempus der Vorvergangenheit ausgedrückt. Ein Tempussystem, das jede zeitliche Abfolge mit einem eigenen Vergangenheitstempus markieren würde, wäre praktisch nicht realisierbar. Neben einem einfachen Vergangenheitstempus müsste es auch ein dazu vorzeitiges Vorvergangenheitstempus, ein dazu wiederum vorzeitiges Vor-Vorvergangenheitstempus usw. geben. Eine Erzählung wie die folgende hätte beispielsweise fünf verschiedene, aufeinander folgende Tempora, wollte man jede zeitliche Abfolge mit einem eigenen Tempus schildern:

(7) Wir suchten im Restaurant-Ratgeber nach passenden Restaurants, wählten eines aus und gingen dorthin. Wenige Minuten später kamen wir am Restaurant an und ließen uns dort das Essen schmecken.

Die hier beschriebenen Ereignisse lassen sich chronologisch wie folgt ordnen:

1. Personen suchen im Restaurant-Ratgeber

2. Wählen ein Restaurant aus

3. Gehen dorthin

4. Kommen dort an

5. Lassen sich das Essen schmecken. 
Tatsächlich werden in Beispiel (7) nicht fünf verschiedene Tempora gebraucht, sondern nur eines (Präteritum), um fünf zeitlich jeweils nacheinander stattfindende Ereignisse darzustellen. Die Frage, in welchen Kontexten (sei es in mündlichen Gesprächen oder in geschriebenen Texten) Vorvergangenheitstempora gebraucht werden, ist also keineswegs trivial.

Grundsätzlich sind es keine speziellen Tempora, sondern konversationelle Implikaturen, durch die der Rezipient die geschilderten Ereignisse in die vom Emittenten gewünschte zeitliche Reihenfolge bringen kann. Levinson erklärt dazu: „when events are conjoined, they tend to be read as temporally successive“ (2000: 122-123). Dementsprechend werden auch ohne eigentliche zeitliche Angaben oder Kontexte Sätze wie, „Hans wrote a novel and he sold the rights to Macmillan“ (2000: 123), in eine zeitliche Reihenfolge gebracht, wonach Hans $z u$ erst den Roman schreibt und ihn danach an Macmillan verkauft. Dass diese Reihenfolge keineswegs im Weltwissen des Rezipienten oder der Lexik der Wörter verankert ist, zeigt sich durch folgende Umformulierungen: „Hans wrote a novel and he sold the rights to Macmillan in advance“ beziehungsweise: „Hans wrote a novel and he sold the rights to Macmillan but not necessarily in that order“ (ebd.). Das Nacheinander in der Erzählzeit impliziert also ein Nacheinander in der erzählten Zeit. Ein Grund hierfür ist auch das Prinzip der chronologischen Erzählung. Diese ist nicht als feste Regel zu begreifen, sondern vielmehr als Absicht des Emittenten, eine Geschichte beziehungsweise ein Ereignis in chronologischer (d. h. ,richtiger') Reihenfolge zu erzählen. Diese Prozessierung des Lesens (beziehungsweise Hörens) vergleicht Partee (1984) mit einer Anapher: „we need to assume that there is a past reference time $r_{p}$ specified at the start of the discourse, and that the introduction of new event sentences moves the reference point forward“ (1984: 254).

Trotz des prinzipiellen Wunsches nach chronologischer Erzählung wird von einer solchen, gerade in Konversationen, immer wieder abgewichen (Kotthoff 2017: 9 und 31-32). Eine solche Divergenz zwischen Erzählzeit und erzählter Zeit ergibt sich, wenn ein nachfolgender Satz (beziehungsweise eine nachfolgende Äußerung) innerhalb der Erzählung vorzeitig zum vorangegangenen ist. Damit der Rezipient, wie oben geschildert, die Ereignisse aber nicht implikatiert als nacheinander, sondern als voreinander begreift, müssen sprachliche Mittel aufgebracht werden, die diese Implikatur aufheben. In obigen Beispielen geschieht dies durch das Adverb in advance beziehungsweise durch den Teilsatz but not necessarily in that order. Ein weiteres Mittel zur Aufhebung der Implikatur liegt in der Verwendung bestimmter Tempora. Dabei wäre es auch möglich, den Satz, „Hans wrote a novel and he sold the rights to Macmillan“, durch die Verwendung des past perfects umzudeuten: „Hans wrote a novel and he [had] sold the rights 
to Macmillan“. Auch ohne eine Vorzeitigkeit anzeigende Subjunktion wie after ist klar, dass Hans die Rechte zuerst verkauft und dann den Roman geschrieben hat. Somit kann ein Tempus der Vorvergangenheit als Reparatur auf metasprachlicher Ebene angesehen werden. Bei diesem wird nicht auf sprachlicher Ebene ein Inhalt durch einen anderen ersetzt, sondern auf metasprachlicher Ebene die Reihenfolge einer Aussage mit einer anderen ausgetauscht.

Neben diesem Aufheben von zeitlichen Implikaturen ist ein Tempus der Vorvergangenheit auch bei sonst ambigen Sätzen und Äußerungen notwendig, um dem Rezipienten eine eindeutige Lesart zu präsentieren. Für das Bairische führt Maiwald hierzu folgendes Beispiel an: „und wenn d'Gluad vagaya war,..., na hod ma oeß mid da grugga ('Krücke') zammkratzt und raus“ (2002: 99). Die Subjunktion wenn könne in diesem Beispiel sowohl der Bedeutung nachdem als auch der Bedeutung während des Standarddeutschen entsprechen. Erst die Verwendung des Plusquamperfekts ließe hier eine eindeutige Lesart zu (Maiwald 2002: 99100). Für das Standarddeutsche lässt sich neben der Subjunktion wenn auch die Subjunktion als anführen. Im Alemannischen findet sich analog dazu häufig die Subjunktion wo mit derselben ambigen Lesart.

\subsection{Aspekt}

\subsubsection{Ist Aspekt eine einzelsprachliche oder übereinzelsprachliche Kategorie?}

In der Forschungsliteratur herrscht keine einhellige Meinung darüber, ob es sich bei Aspekt um eine nur in manchen Sprachen vorhandene Kategorie handelt, die durch Flexion zum Ausdruck kommt, oder um eine übereinzelsprachliche, die in unterschiedlichen Einzelsprachen verschiedene Realisierungsmöglichkeiten kennt. Daraus ergeben sich weitere Unterschiede - etwa bei den Fragen, wo der Unterschied zwischen Aktionsart und Aspekt zu sehen ist und welche Aspektklassifizierungen möglich sind. Ich möchte daher zunächst die zwei Lager aufzeigen, in die die Aspektforschung eingeteilt werden kann.

Die eine (konservative) Seite, zu der vor allen Dingen viele Slawisten (etwa Admoni 1982), aber auch Indologen (z. B. Nespital 1983) zählen, beschränkt Aspekt auf Sprachen, bei denen dieser als sechste Verbkategorisierung (neben Person, Numerus, Tempus, Modus und Genus Verbi) vorhanden ist und morphologisch eindeutig von den anderen fünf Kategorien unterschieden werden kann (Thieroff 1992: 65). Wesentlich für diese Gruppe ist eine strenge Aspektklassifizierung in die Opposition perfektiv/imperfektiv. Nespital beispielsweise bestreitet für Sprachen, die nicht über diese strenge Opposition verfügen, die Existenz eines Aspektsystems und sieht bei den Nicht-Aspektsprachen in dieser Hinsicht 
„ein durch nichts kompensierbares semantisches Defizit“ (Nespital 1983: 375), das also nicht durch Verbformen oder das Hinzuziehen anderer kontextueller Mittel ausgeglichen werden kann (ebd.). Dabei spricht er sich klar gegen eine Ausweitung der Aspektklassifizierung aus. Aspekt habe einen spezifischen Aussagewert über die in der Slawistik gebrauchte grammatische Opposition. Die Verwendung von Aspekt als ,diffuser Oberbegriff [...] mit Bezug auf alle möglichen aktionalen und temporalen Merkmale und Oppositionen völlig unterschiedlicher grammatischer Verbformen“ (Nespital 1983: 359) würde Aspekt seines spezifischen Aussagewerts berauben.

Vertreter dieser Forschungsrichtung betrachten als Mittel zum Ausdruck von Aspekt also ausschließlich unterschiedliche morphologische Formen desselben Verbs. Aktionsartliche Unterschiede, wie sie sich beispielsweise im Standarddeutschen bei der Wortbildung von Verben wie jagen zu erjagen ergeben, sind dagegen nicht als Aspekt aufzufassen, da diese Art, Abgeschlossenheit auszudrücken, nicht reihenbildend geschieht, sondern auf einige wenige Verben beschränkt ist.

Die andere (modernere) Seite der Aspektforschung fasst den Aspektbegriff weniger eng und betrachtet ihn als übereinzelsprachliche Kategorie, die verschiedene morphologische, morphosyntaktische oder lexikalische Ausdrucksmittel kennt (vgl. dazu u. a. Lyons 1971, Comrie 1981 und Bybee 1985). Dabei wird das slawische Aspektsystem nicht als prototypisch, sondern eher als untypisch (Bybee/Dahl 1989: 80) verstanden. Dahl stellt sogar in Frage, ob dessen feste Aspektopposition überhaupt den übereinzelsprachlichen Kategorien perfektiv und imperfektiv entspricht (vgl. Dahl 1985: 85). Kennzeichnend für das Vorhandensein einer grammatischen Kategorie Aspekt in einer Sprache ist nach dieser Auffassung, dass lexikalische, morphologische oder morphosyntaktische Mittel systematisch als Aspektmarkierungen gebraucht werden können (Maiwald 2002: 21). Die Untersuchungen von Bybee (1985) und Dahl (1985) legen den Schluss nahe, dass der Großteil der von ihnen betrachteten Sprachen über eine grammatische Kategorie Aspekt verfügt und diese morphologisch sogar häufiger realisiert wird als die Kategorie Tempus (Leiss 1992: 27).

Bei der Frage, wie weit oder eng der Begriff Aspekt anzusehen ist, fällt häufig der auf Bondarko (1967) zurückgehende Begriff der Aspektualität (vgl. dazu u. a. Rödel 2007). Bondarko (1967) versteht unter Aspektualität eine Kategorie, die den zeitlichen Verlauf einer Situation beschreibt und durch unterschiedliche Mittel zum Ausdruck kommt. Hierzu zählen unter anderem morphologische, lexikalische und kontextuelle Mittel (Schmid 2014: 37-38). Damit fasst dieser Begriff nicht nur Aspekt und Aktionsart zusammen, sondern erlaubt auch eine kategorische Erfassung häufig als frei und unfest empfundener kontextueller Mittel. Aus 
diesem Grund wird Aspektualität von vielen als eine Art Kompromiss zwischen den oben beschriebenen Positionen angesehen und gerade bei der Untersuchung sogenannter aspektloser Sprachen wie dem Deutschen herangezogen (vgl. u. a. Henriksson 2006).

Für die Untersuchung der Vergangenheitstempora des Alemannischen wird in dieser Arbeit allerdings nicht der Begriff der Aspektualität verwendet, da ich überprüfen möchte, ob Aspekt im Deutschen beziehungsweise Alemannischen als grammatische Kategorie in Form von Tempus-Variation existiert. Ich schließe mich dabei den Ansichten Lyons (1971), Comries (1981) und Bybees (1985) an und betrachte Aspekt als übereinzelsprachliche grammatische Kategorie, die in den jeweiligen Einzelsprachen teils unterschiedliche, aber systematische Realisierungsmöglichkeiten kennt. Flexion betrachte ich demzufolge nicht als notwendiges und hinreichendes Kriterium, um von Aspekt sprechen zu können. Im Folgenden soll daher zunächst eine übereinzelsprachlich anwendbare Definition von Aspekt vorgestellt werden, um davon in Kapitel 2.3 die Kategorie Aktionsart abzugrenzen.

\subsubsection{Definition}

Wie bereits eingangs dieses Kapitels erwähnt, handelt es sich bei Aspekt um eine grammatische Kategorie, die den inneren zeitlichen Verlauf eines Geschehens zum Ausdruck bringt (Comrie 1981: 3). Vereinfacht gesagt, unterscheidet der Sprecher je nach ausgedrücktem Aspekt, ob das dargestellte Ereignis als Ganzes (=perfektiver Aspekt) oder als sich im Verlauf befindend (=imperfektiver Aspekt) betrachtet wird. Beim Aspekt geht es also um die vom Sprecher eingenommene Perspektive zum Geschehen: Steht er außerhalb der Situation und überblickt diese somit als Ganzes oder befindet er sich inmitten der Situation und kann deren Grenzen nicht erblicken (vgl. dazu Isačenko 1962: 348)? Vom Tempus lässt sich diese Kategorie dabei durch die nicht-zeitlich-deiktische Funktion unterscheiden.

Das weitere Kapitel 2 verdeutlicht Aspekt anhand aus der Introspektion gewonnener standarddeutscher Beispiele. Hierbei verwende ich zur Beschreibung der Verbalsituationen die Begriffe perfektiv und imperfektiv. Dabei soll aber nicht postuliert werden, das Standarddeutsche besitze eine grammatische Kategorie Aspekt. Voraussetzung dafür wäre eine systematische Markierung abgeschlossener und unabgeschlossener Situationen durch lexikalische, morphologische oder morphosyntaktische Mittel, von der ich in dieser Arbeit nicht ausgehe. Die 
Verwendung standarddeutscher Beispielsätze dient lediglich der besseren Veranschaulichung.

Obwohl die Auffassung, dass Aspekt eine nicht-zeitlich-deiktische Funktion hat, gemeinhin Zuspruch findet, wird der Kategorie Aspekt häufig der Ausdruck des Verhältnisses zwischen Ereigniszeit und Referenzzeit zugeschrieben (vgl. hierzu u. a. Klein 1994: 99, Schlegel 2000: 60, Maiwald 2002: 101, Musan 2002: 6 und Gillmann 2016: 44-46) und vom Tempus dadurch unterschieden, dass letzteres das Verhältnis von Sprechzeit zu Referenzzeit ausdrückt. Diese Annahme ist wohl nicht als Widerspruch zur nicht-deiktisch-zeitlichen Funktion von Aspekt zu betrachten, sondern ergibt sich sekundär über den abgeschlossenen beziehungsweise nicht-abgeschlossenen Zustand eines durch den perfektiven beziehungsweise imperfektiven Aspekt ausgedrückten Ereignisses. Das meint, dass ein Ereignis, das in der Vergangenheit abgeschlossen ist, notwendigerweise zu einer Zeit vor der Referenzzeit stattgefunden haben muss; wohingegen ein Ereignis, das in der Vergangenheit unabgeschlossen ist, während der Referenzzeit stattfinden muss. Vertreter dieser Aspektauffassung setzen also Vorvergangenheit mit abgeschlossen in der Vergangenheit und Vergangenheit mit unabgeschlossen in der Vergangenheit gleich. Diese Ansicht lässt sich folgendermaßen verdeutlichen: 


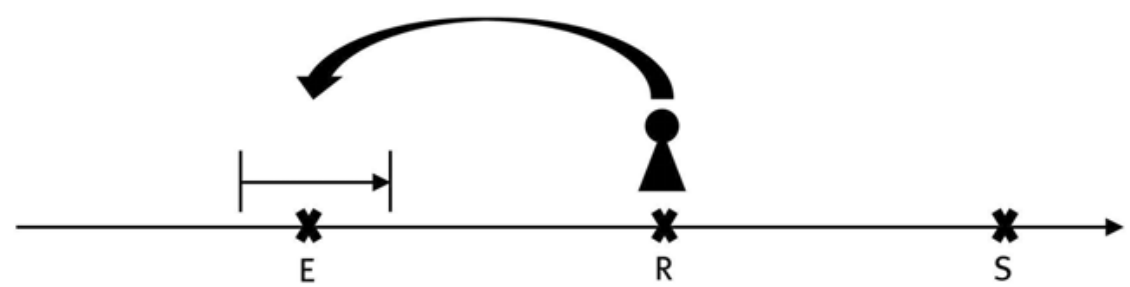

„Nachdem sie das Essen gekocht hatte, servierte sie es.“ 'Gegenwart”

Abb. 1: Perfektiver Aspekt als E vor R

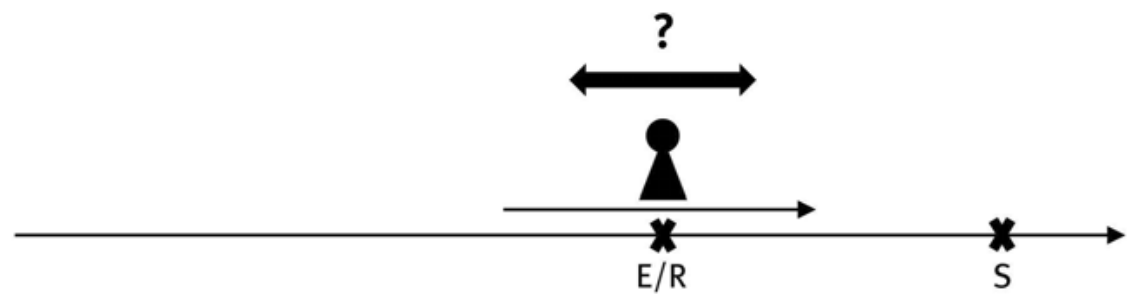

„Sie hörte Musik, während sie kochte.“ 'Gegenwart'

Abb. 2: Imperfektiver Aspekt als $E=R$

Der Sprecher blickt in beiden Beispielsätzen von der Referenzzeit aus auf ein weiteres Ereignis. Während dies in Abbildung 1 bereits abgeschlossen ist, dauert es in Abbildung 2 zum Zeitpunkt des Betrachtens noch an. Der Sprecher ist also im ersten Fall in der Lage, die Situation von außen zu betrachten und somit als Ganzes zu beurteilen. Im zweiten Fall befindet er sich dagegen inmitten der Situation und ist außerstande, die Grenzen der Situation zu erfassen und kann das Ereignis daher nur als sich im Verlauf befindend beschreiben. Aus dieser Sprecherperspektive ergebe sich erst das Verhältnis von $\mathrm{E} z \mathrm{z} R$ als vorzeitig beziehungsweise gleichzeitig. Während die grammatische Kategorie Tempus einen direkten Verweis von $\mathrm{S}$ zu $\mathrm{R}$ herstelle, ergebe sich die Relation von $\mathrm{E} \mathrm{zu} \mathrm{R}$ nicht als deiktischer Verweis, sondern als implizite Folge der (Un-)Abgeschlossenheit. Für diese Ansicht ließe sich auch die Tatsache anführen, dass (im Verständnis der traditionellen Slawistik) ,klassische‘ Aspektsprachen wie das Russische über kein ausdifferenziertes Tempussystem verfügen, das zwischen Vergangenheit und Vorvergangenheit unterscheiden kann. Stattdessen wird im Russischen das 
zeitliche Voreinander mit Hilfe des perfektiven Aspekts ausgedrückt (vgl. Appel 1996: 9).

Dagegen lässt sich allerdings einwenden, dass das Russische, wie bereits beschrieben, (nach allgemeinlinguistischen Ansätzen) keine typische Aspektsprache darstellt. Somit kann das Russische, das eine Unterspezifizierung bei der Verbkategorie Tempus durch die Kategorie Aspekt kompensiert, auch nicht als Paradebeispiel für das Zusammenspiel von Tempus und Aspekt dienen.

Ich widerspreche in dieser Arbeit der Ansicht, dass Vorvergangenheit mit Abgeschlossenheit in der Vergangenheit und einfache Vergangenheit mit Unabgeschlossenheit in der Vergangenheit identisch seien. Gegen diese Auffassung von Aspekt lassen sich im Wesentlichen zwei Argumente anführen. Zum einen führt die eben beschriebene Gleichsetzung zu einer Unterspezifizierung; zum anderen widerspricht die zeitliche Verortung von perfektivem und imperfektivem Aspekt als ein Nacheinander dem so genannten Inzidenzschema, das, wie Thieroff (1992: 68) konstatiert, in beinahe allen Arbeiten zum Aspekt als notwendige Funktion des Aspekts angeführt wird.

Setzt man Vorvergangenheit und Abgeschlossenheit in der Vergangenheit auf die oben beschriebene Weise gleich, so impliziert das, dass jede Situation, die vor der Referenzzeit beginnt, auch vor dieser endet. Denn nur eine zur Referenzzeit abgeschlossene Situation kann der Sprecher sozusagen ,von außen“ betrachten. Dass aber nicht jede in der Vorvergangenheit situierte Situation zur Referenzzeit (also in der einfachen Vergangenheit) abgeschlossen ist, zeigen folgende Beispielsätze (8).

(8) a. Er hatte Fußball schon gemocht, [bevor seine Mannschaft das Finale erreichte, und nun freute er sich auf das große Spiel.]

b. Sie war schon einige Zeit Beamtin gewesen, [als ihr gekündigt werden sollte.]

c. Der Schnee war schon lange gelegen, [als sie sich entschloss, ihn wegzuräumen.]

d. Der Mann war schon vorher jeden Morgen zu spät in die Arbeit gekommen; [der zerstörte Wecker änderte daran dann auch nichts mehr.]

In allen vier Beispielsätzen wird ein Ereignis beziehungsweise ein Zustand beschrieben, dessen nicht genau terminierter Beginn vor der Referenzzeit angesetzt wird und gleichzeitig über die Referenzzeit hinausreicht. In Beispiel (8a) wird betont, dass eine Person schon vor dem Finaleinzug ihres Teams Fußball mochte. Zur Referenzzeit, also dem sich-Freuen auf das große Spiel, dauert dieser Zustand 
noch an. Von abgeschlossenen Zuständen kann auch in den anderen Sätzen nicht die Rede sein. So ist die Beamtin in Beispiel (8b) auch zur Referenzzeit, als man ihr kündigen möchte, noch Beamtin (und somit auch vor der Kündigung geschützt). Zu welchem Zeitpunkt der Schnee aus Beispiel (8c) nicht mehr liegt, ist, ausgehend von diesem Beispielsatz, nicht ersichtlich; zumindest zum Zeitpunkt des Entschlusses, ihn wegzuräumen, und (mindestens) kurze Zeit darauf, liegt er aber noch. Das in Beispiel (8d) genannte sich wiederholende Ereignis des Zuspätkommens reicht sogar ganz explizit von einem Zeitpunkt aus der Vorvergangenheit über die Referenzzeit, die Zerstörung des Weckers, hinaus. Graphisch lassen sich die vier Beispielsätze folgendermaßen darstellen:

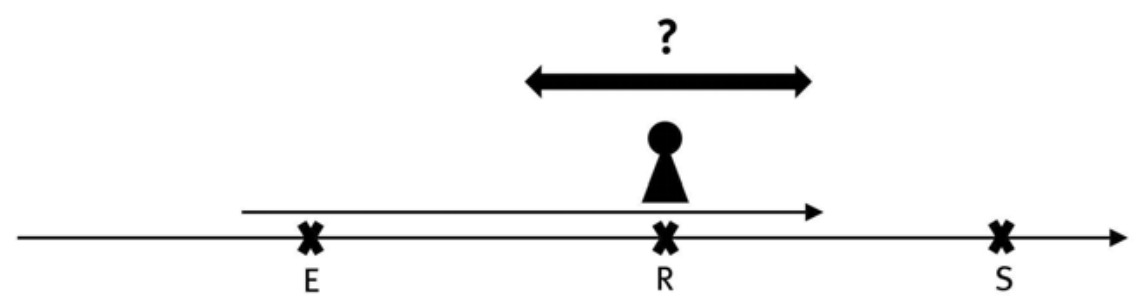

Abb. 3: Imperfektiver Aspekt als E vor R

Dass E vor R liegt, wird hier im Gegensatz zur obigen Illustration explizit gemacht. Genau wie im obigen Beispiel, das Unabgeschlossenheit in der Vergangenheit verdeutlichen soll, reicht die Zeitspanne $\mathrm{E}$ aber über $\mathrm{R}$ hinaus.

An dieser Stelle lohnt auch ein Blick auf das Englische, dessen progressive in fast allen Arbeiten als Aspekt anerkannt wird (vgl. Thieroff 1992: 69). Im Englischen ist es also möglich, in verschiedenen Zeitstufen jeweils zwischen einer progressive- und einer nicht-progressive-Form zu alternieren. Dass für die Tempusformen simple past, present perfect simple und past perfect simple je eine progressive-Form existiert, zeigt, dass eine Gleichsetzung von perfektivem Aspekt und den Tempora der Vorvergangenheit grundlegend abzulehnen ist. Betrachtet man perfektiven Aspekt und Tempora der Vorvergangenheit als identisch, spricht man dem ausdifferenzierten Tempussystem einer Sprache dessen spezielle Funktion zur Markierung zeitlicher Verhältnisse ab. Ob allerdings das Alemannische über ein derartiges kombiniertes Tempus-Aspekt-System verfügt, muss erst noch gezeigt werden. 
Gravierender als diese Unterspezifizierung ist jedoch der Verstoß gegen das Inzidenzschema, den die Gleichsetzung von Vorvergangenheit und perfektivem Aspekt der einfachen Vergangenheit nach sich zieht. Das von Pollak erstmals so genannte „Inzidenzschema“ (1960: 129) wird in fast allen Arbeiten, die Aspekt als übereinzelsprachliche Kategorie begreifen und sich theoretisch und grundlegend mit Aspekt auseinandersetzen, als hinreichendes Kriterium angesehen, um einer Sprache ,Aspektrelevanz‘ bescheinigen zu können (vgl. Thieroff 1992: 69). Es beschreibt das Zusammenspiel von Perfektivität und Imperfektivität und bildet damit die eigentliche Funktion von Aspekt ab. Klein (1974) fasst die Funktion des Inzidenzschemas folgendermaßen zusammen:

\begin{abstract}
Das mit dem imperfektiven Aspektmorphem versehene Verb (Imparfait) liefert in seiner Prozessualität gewissermaßen die Handlungs- oder Geschehensbasis für eine perfektiv (Passé composé oder simple) gekennzeichnete Handlung, die durch syntaktische Verknüpfung mit der Basishandlung inzidiert: Inzidenz. Eine Sprache, die zwei morphologisch differenzierte Formen zur Zeitstufendifferenzierung für jedes Verb besitzt, die in einem solchen Schema korrelativ benutzt werden können, ist als aspektrelevant zu bezeichnen
\end{abstract}

(Klein 1974: 82).

Ein unabgeschlossenes (also nicht in seiner Ganzheit erfasstes) Ereignis dient also als Handlungsrahmen, in das ein abgeschlossenes (also in seiner Ganzheit erfasstes) Ereignis situiert wird. Comrie listet zur Veranschaulichung des Wirkens von imperfektivem und perfektivem Aspekt folgenden (inhaltlich identischen) Satz für das Englische, das Russische, das Französische, das Spanische und das Italienische (Comrie 1981: 3):

\begin{tabular}{|c|c|c|c|c|}
\hline English: & John & was reading & when I & entered. \\
\hline Russian: & Ivan & čital, & kogda ja & vošel. \\
\hline French: & Jean & lisait & quand & $\mathrm{j}$ 'entrai. \\
\hline Spanish: & Juan & leía & cuando & entré. \\
\hline Italian: & Gianni & $\begin{array}{l}\text { leggeva } \\
\text { IVerb } 1\end{array}$ & quando & $\begin{array}{l}\text { entrai. } \\
\text { Verb 21 }\end{array}$ \\
\hline
\end{tabular}

Ohne den Begriff des Inzidenzschemas zu verwenden, liefert er eine Beschreibung, die sich beinahe vollständig mit der von Klein (1974) deckt:

In each of these sentences, the first verb presents the background to some event, while that event itself is introduced by the second verb. The second verb presents the totality of the situation referred to (here, my entry) without reference to its internal temporal constituency: the whole of the situation is presented as a single unanalysable whole, with beginning, middle, and end rolled into one; no attempt is made to divide this situation up into the various individual phases that make up the action of entry. Verbal forms with this 
meaning will be said to have perfective meaning, and where the language in question has special verbal forms to indicate this, we shall say that it has perfective aspect.

(Comrie 1981: 3)

Die Frage, ob das Ereignis im perfektiven Aspekt das Ereignis im imperfektiven Aspekt unterbricht oder nicht, ist dabei kein entscheidendes Kriterium für das Vorhandensein von Aspekt. In manchen Fällen bleibt sie überhaupt unbeantwortet (Pollak 1960: 130). Demzufolge ist es, bezogen auf obigen Beispielsatz, irrelevant, ob John/Ivan etc. mit dem Lesen aufhört, als das Ich des Textes den Raum betritt. Für die Bewertung einer Situation als perfektiv ist darüber hinaus nicht relevant, ob das beschriebene Ereignis sich über eine kurze oder längere Zeitdauer erstreckt oder nur punktuell (wie in obigem Beispielsatz) stattfindet. Entscheidend ist, dass es als Ganzes wahrgenommen und nicht dessen Verlauf beschrieben wird. Daher lässt sich auch ein Satz wie (9) dem Inzidenzschema zuordnen:

(9) Während ich in Freiburg wohnte, besaß ich für zwei Jahre einen Hund.

Auch hier wird das holistische Ereignis des Hundebesitzens in das unabgeschlossene Ereignis des In-Freiburg-Wohnens situiert und kann deshalb als perfektiv betrachtet werden. Diese Aspektfunktion existiert dabei nicht nur innerhalb eines Satzes (in Haupt- und Nebensatz), sondern kann sich über einen größeren Kontext oder den gesamten Text erstrecken, wie in (10) deutlich wird.

(10) Während ich in Freiburg wohnte, sind mir immer wieder schlimme Dinge widerfahren. Einmal wurde mir das Handy gestohlen. Ein anderes Mal wurde ich von einem Auto angefahren...

Hier liefert der Teilsatz, Während ich in Freiburg wohnte, die Rahmenhandlung, in die verschiedene perfektive Handlungen situiert werden. Die Rahmenhandlung reicht in diesem Beispiel über die Satzgrenze hinaus, sodass auch die perfektiven Ereignisse der nachfolgenden Sätze in die imperfektive Situation des InFreiburg-Wohnens situiert werden können.

Eine perfektive Verbalhandlung muss auch nicht gezwungenermaßen im Handlungsrahmen einer imperfektiven Verbalhandlung stehen. Sie muss aber zumindest theoretisch um einen solchen Handlungsrahmen ergänzt werden können.

(11) Letzten Dienstag ist er mit dem Fahrrad gestürzt. 
Dieser singuläre Satz (11) ist perfektiv, da er ein Ereignis als abgeschlossen und ganzheitlich darstellt. Eine imperfektive Situation, die den Handlungsrahmen bildet, könnte folgendermaßen ergänzt werden:

(12) Letzten Dienstag [war er auf dem Heimweg und] ist mit dem Fahrrad gestürzt.

Die Gleichsetzung von perfektivem Aspekt in der Vergangenheit und temporaler Vorvergangenheit verstößt insofern gegen das Inzidenzschema, als die perfektive Situation niemals in die imperfektive hingesetzt werden kann, da erstere zu Beginn von zweiterer bereits abgeschlossen und beendet ist. Somit ist die Identifizierung von Vorvergangenheit und perfektivem Aspekt der einfachen Vergangenheit abzulehnen.

\subsubsection{Das Spannungsfeld von Tempus und Aspekt}

Wie bereits erläutert, muss eine Situation, um sie in ihrer Ganzheit perspektivieren zu können, in der darauffolgenden Zeitstufe (d. h. bei einer perfektiven Situation in der Vorvergangenheit zu einem Zeitpunkt in der Vergangenheit und bei einer perfektiven Situation in der Vergangenheit zu einem Zeitpunkt in der Gegenwart) abgeschlossen und beendet sein. Die Referenzzeit muss also der Ereigniszeit nachgeordnet sein. Diese Tatsache verleitet häufig zur Übergeneralisierung, eine sich im Verlauf befindende Verbhandlung dürfe in der darauffolgenden Zeitstufe nicht abgeschlossen und beendet sein. Betrachtet man einen Satz wie (13) mit einem Verb im Tempus Perfekt als perfektiv, so darf die Verbhandlung zur Sprech- und Referenzzeit (wenn man die beiden Zeiten beim Tempus Perfekt als gleichzeitig anordnet) keine Gültigkeit mehr besitzen.

(13) Früher habe ich den Film gut gefunden.

Die Aussage müsste also um einen Zusatz wie, mittlerweile mag ich ihn nicht mehr, erweitert werden können. Ebenso scheinen Sätze wie (14) die These zu belegen, dass imperfektive Situationen in die nächste Zeitstufe hineinreichen.

(14) a. Ich hatte nie ein Problem damit und habe immer noch keines.

b. Ich fand, dass der Film gut ist, und finde es noch. 
Und tatsächlich können imperfektive Verbhandlungen in ebensolcher Weise gebraucht werden. Entscheidend ist jedoch, dass die Begrenzung kein zwingendes Unterscheidungskriterium für die Aspektopposition ist. Klein bemerkt hierzu, dass die Abgeschlossenheit (zur nächsten Zeitstufe) bei perfektiv impliziert, bei imperfektiv jedoch nicht ausgeschlossen sei (1974: 79). Bei der Beurteilung nach Perfektivität ist Abgeschlossenheit zur nächsten Zeitstufe also ein notwendiges, aber kein hinreichendes Kriterium. Das hinreichende Kriterium für die Unterscheidung von perfektivem und imperfektivem Aspekt ist das mögliche Vorkommen im bereits eingeführten Inzidenzschema. ${ }^{10}$

Dementsprechend erfolgt bei den nachfolgenden Teilsätzen in (15) eine $\mathrm{Zu}$ weisung zum imperfektiven Aspekt, obwohl die darin beschriebenen Situationen zur Sprechzeit bereits abgeschlossen sind:

(15) a. Während die Senatoren debattierten, [überquerte Cäsar den Rubikon.]

b. Als Bobby Fischer auf dem Weg zum Brett war, [machte Boris Spassky den ersten Zug.]

Obwohl die Verbalhandlungen in beiden Teilsätzen zur Sprechzeit bereits abgeschlossen sind, lassen sie sich zweifelsfrei dem imperfektiven Aspekt zuordnen. Entsprechend der Funktion des Inzidenzschemas setzen die beiden Teilsätze den Handlungsrahmen, in den ein einzelnes abgeschlossenes Ereignis situiert wird. Indem Aspekt „foreground (i. e. the main narrative line or principal statements) and background [Hervorhebungen im Original] (the subsidiary, supportive material) of discourse“ (Binnick 2001: 562) unterscheidet, erfüllt er diskursstrukturierende Funktion (vgl. dazu auch Hopper 1979: 213-215, Bybee et al. 1994: 126, Rothstein 2007: 71-82 und Fischer 2018: 191). Inwiefern auch Tempora diskursstrukturierende Funktion einnehmen können, möchte ich in Kapitel 4.3.5 aufzeigen.

Fassen wir noch einmal zusammen: Ich definiere Aspekt als die Perspektive, die ein Sprecher zu einer beschriebenen Situation einnimmt. Er stellt diese dabei entweder als ganzheitlich (=perfektiv) oder als sich im Verlauf befindend (=imperfektiv) dar. Tempus dagegen besitzt eine deiktische Funktion, indem es von einer Zeitstufe auf eine andere verweist. Die beiden Kategorien überschneiden

10 Ich werde die Begriffe perfektiv und imperfektiv in den Analysekapiteln vermeiden, da diese eine grammatische Kategorie Aspekt implizieren, die noch nachzuweisen wäre. Um terminologische Unklarheiten auszuschließen, werde ich stattdessen die Begriffe abgeschlossen und unabgeschlossen verwenden. Damit ist nicht die (Un-)Abgeschlossenheit zur nächsten Zeitstufe gemeint, sondern die intrinsische (Un-)Abgeschlossenheit. 
sich teilweise aufgrund von Implikaturen, sind aber konzeptuell eigenständig zu betrachten. Ein Sprecher kann also auf verschiedene Zeitstufen verweisen und jeweils Situationen als abgeschlossen oder als unabgeschlossen beschreiben.

Um Situationen als ganzheitlich beschreiben zu können, muss der Sprecher außerhalb dieser Situationen stehen. Das heißt, die Referenzzeit (als Bezugspunkt der Beschreibung) darf nicht zeitgleich sein mit der Ereigniszeit. Allerdings nimmt ein Sprecher nicht immer eine perfektive Perspektive ein, wenn die Referenzzeit der Ereigniszeit nachgeordnet ist. Die Nachordnung der Referenzzeit zur Ereigniszeit ist dementsprechend die Voraussetzung für den perfektiven Aspekt, aber kein hinreichendes Kriterium. Obwohl das Verhältnis von Ereignis- und Referenzzeit der Kategorie Tempus zugeschrieben werden muss, hat es also auch für Aspekt eine wichtige Bedeutung. Hier zeigt sich die enge Verbindung der beiden Kategorien: Indem das Tempus die drei Reichenbach'schen Zeiten anordnet, legt es fest, wo der perfektive und imperfektive Aspekt ausgedrückt werden können. Dies zeigt sich beispielsweise beim Präsens mit perfektivem Aspekt, da eine perfektive Darstellung einer gegenwärtigen Situation nicht möglich ist. Ein Satz wie (16), der aufgrund des nicht-durativen telischen Verbs gewinnen (Achievement) eine perfektive Bedeutung hat und im Präsens steht, muss daher als zukünftig aufgefasst werden, d. h. in diesem Satz verweist der Sprecher nicht auf die aktuelle Situation, sondern auf eine zukünftige. Die Ereigniszeit ist also nach der Referenzzeit, weshalb eine perfektive Darstellung möglich ist (vgl. hierzu auch Henriksson 2006: 86).

(16) Ich gewinne.

Am einfachsten lässt sich das Zusammenspiel von Tempus und Aspekt anhand vergangener und vorvergangener Situationen erklären. Bei Tempora der einfachen Vergangenheit (Präteritum und Perfekt) verweist der Sprecher auf einen Punkt in der Vergangenheit. Drückt er zusätzlich den perfektiven oder imperfektiven Aspekt aus, so situiert er ein perfektives Ereignis in einen imperfektiven Bezugsrahmen. Dies wird im folgenden Satz (17) deutlich.

(17) Während die Wachen schliefen [=imperfektiver Handlungsrahmen], ist er entkommen [=perfektives Ereignis].

Bei einem Tempus der Vorvergangenheit (Plusquamperfekt) verweist der Sprecher auf einen Punkt vorzeitig zu einem in der Vergangenheit liegenden Punkt. Auch hier kann ein perfektives Ereignis in einen imperfektiven Handlungsrahmen situiert werden. 
(18) Zwei Jahre saß er im Gefängnis [=einfache Vergangenheit]. Während er noch ein freier Mann gewesen war [=Vorvergangenheit und imperfektiver Handlungsrahmen], hatte er einmal viel Geld beim Glücksspiel gewonnen [=Vorvergangenheit und perfektives Ereignis].

Hier wird deutlich, dass Tempus und Aspekt zwar einander greifen, aber eigenständige Bedeutungen haben.

\subsection{Aktionsart}

\subsubsection{Definition}

In Abgrenzung zur grammatischen Kategorie Aspekt betrachte ich Aktionsart als eine lexikalisch-semantische Kategorie, die die Verlaufsweise einer Situation, also eine dem Vorgang objektiv inhärente Charakteristik, festlegt. Diese lexikalisch-semantische Auffassung von Aktionsart findet sich unter anderem auch bei Erben (1980), Steinitz (1981), Wierzbicki (1999), Li/Shirai (2000), Helbig/Buscha (2001), Nicolay (2007) und Heinold (2015). Während sich die Ansichten der genannten Autoren im Hinblick auf den lexikalisch-semantischen Charakter decken, unterscheiden sich die Meinungen bei der Frage, wie und an welcher Stelle die Aktionsart zum Ausdruck kommt. So vertritt Erben die Meinung, die Aktionsart könne auch durch morphologische und syntaktische Bildungen gekennzeichnet werden (1980: 72-74). Wierzbicki dagegen sieht die Aktionsart bereits in der Lexik verankert und extern hinzutretende Realisierungen als Teil der grammatischen Kategorie Aspekt:

\footnotetext{
Wie ein Ereignis dargestellt werden kann, als dauerhaft oder zeitlich punktuell, als abgeschlossen oder zeitlich offen, ist Gegenstand der Perspektive. Lexikalisch wird die Perspektive durch die Aktionsarten ausgedrückt und grammatisch durch den Aspekt, wobei die Aktionsarten eine konventionalisierte Perspektive wiedergeben und der Aspekt die aktuelle Perspektive realisiert
}

(Wierzbicki 1999: 10).

Ich folge in meiner Arbeit der Ansicht Wierzbickis, wobei ich auch die Wortbildung als Teil der Lexik und somit als Mittel zur Markierung der Aktionsart betrachte.

Nicolay weist - genau wie Vendler (1967: 102-103) und Henriksson (2006: 45-46) - einzelnen Verben in Anlehnung an Krifka (1989: 41) und Bäuerle (1994: 4-5) je nach Vorhandensein oder Nicht-Vorhandensein von Ergänzungen die eine oder andere Aktionsart zu (Nicolay 2007: 8). Während essen als Prozess 
angesehen wird, bezeichnet sie einen Apfel essen als Ereignis (ebd.). Diese Unterscheidung betrifft aber nicht die Aktionsart, sondern eine unterschiedliche Gesamtperspektive. Aktionsartlich gesehen bleibt die durch das Verb essen ausgedrückte Handlung identisch. Es ändert sich allerdings die Perspektive des Sprechers auf die bloße Handlung des Essens oder auf das holistische Ereignis des Apfelessens. Im Gegensatz zur Aktionsart ist diese Darstellung aber nicht in der Lexik verankert und somit auch nicht objektiv, sondern drückt die subjektive Betrachtung des Sprechers aus. Der einzelne Sprecher kann also, anders als bei der spezifischen Verbbedeutung, die in der Aktionsart erfasst wird, durch grammatische Formen, Adverbien und Ergänzungen oder syntaktische Konstruktionen die Abgeschlossenheit oder Unabgeschlossenheit einer Situation ausdrücken. Ich widerspreche damit der Ansicht Nicolays, die Bezug nehmend auf Verkuyl (1989), Krifka (1989) und Egg (1994) den Geltungsbereich der Aktionsart nicht nur auf Verblexeme, sondern auch auf Verbalphrasen und sogar ganze Sätze bezieht (vgl. Nicolay 2007: 8).

Während die Aktionsart die einzelne Situation separiert vom Kontext beschreibt, liefert der Aspekt eine Beschreibung des Gesamtkontextes der Situation. Dies lässt sich am besten anhand von Beispielen erläutern.

(19) a. Nur einmal haben wir zusammen gegessen.

b. Jeden Abend $a \beta$ das Kind sein Gemüse auf.

c. Sie nießte für einige Zeit. [Es schien, als wollte sie gar nicht mehr damit aufhören.]

d. Sein Freund wusste immer die richtigen Antworten auf die Fragen der Lehrer.

Die in diesen Beispielen (19) verwendeten Verben zur Beschreibung von Situationen drücken verschiedene Aktionsarten aus. Der Aspekt dagegen wird nicht mit Hilfe der spezifischen Verbsemantik, sondern durch Adverbien (nur einmal, jeden Abend etc.) vermittelt. Der Vorgang des Essens in Beispiel (19a) ist durativ, dynamisch und atelisch (also der Aktionsart Activity zuzuordnen); das gesamte Ereignis dagegen wird auf einen einzigen abgeschlossenen Zeitraum festgelegt und daher zum Aspekt perfektiv gerechnet. Das Verb in Beispiel (19b) beschreibt zwar einen Vorgang, der auf einen Kulminationspunkt zuläuft; der gesamte Satz dagegen drückt eine längere Phase aus und damit den imperfektiven Aspekt. Konkret heißt das für das Beispiel (19b), dass der eigentliche Vorgang des Aufessens zwar nur einmal stattfindet (was durch die Aktionsart ausgedrückt wird), aber über eine Phase hinweg immer wieder geschieht (was durch den Aspekt vermittelt wird). Demgegenüber drückt das Verb in Beispiel (19c) ein einmaliges 
nicht-duratives Ereignis aus. Der Sprecher perspektiviert allerdings die Verlaufsweise für einige Zeit und somit den imperfektiven Aspekt. Beschrieben wird also der unabgeschlossene Vorgang des Nießens, der aus vielen Einzelereignissen besteht. Neben den Beispielen (19a-c), in denen sich die zeitlichen Verlaufsweisen von der Aktionsart des Verbs und dem Aspekt des Satzes entgegenstehen, können sie sich natürlich auch wie in Beispiel (19d) decken. Dort vermitteln sowohl die spezifische Verbsemantik als auch der durch den Kontext geschaffene Aspekt einen andauernden Zustand.

Ich vertrete in dieser Arbeit die Meinung, dass in kontextlosen Sätzen die Aktionsart die Gesamtperspektive anzeigt. Hier wird die Schnittstelle zwischen Aktionsart und Aspekt deutlich. Während die durativen und nicht-grenzbezogenen (=atelischen) Aktionsarten Activity und State ohne weiteren Kontext auf unabgeschlossene Situationen hindeuten, signalisieren die Aktionsarten Accomplishment, Achievement und Semelfactive abgeschlossene Situationen. Dementsprechend kann jede Situation als abgeschlossen oder unabgeschlossen kategorisiert werden (vgl. Kap. 3.2.3).

Nicht nur die eben beschriebenen Probleme bei der theoretischen und praktischen Abgrenzung der Aktionsart von Aspekt, sondern auch die unterschiedlichen Ansichten zur lexikalisch-semantischen Reichweite von Aktionsart sorgen für Unklarheit. Helbig/Buscha (2001) definieren die Bedeutungsreichweite von Aktionsarten beispielsweise folgendermaßen:

Unter der Aktionsart eines Verbs versteht man die Verlaufsweise und Abstufung des Geschehens, das vom Verb bezeichnet wird. Die Differenzierung des Geschehens erfolgt nach dem zeitlichen Verlauf (Ablauf, Vollendung; Anfang, Übergang, Ende) und nach dem inhaltlichen Verlauf (Veranlassung, Intensität, Wiederholung, Verkleinerung). Der zeitliche und inhaltliche Verlauf greifen oft ineinander

(Helbig/Buscha 2001: 62).

Bei dieser Kategorisierung ergeben sich jedoch bei näherer Betrachtung einige Probleme. Fraglich ist, ob sich die unterschiedlichen Aktionsklassen überhaupt miteinander vergleichen lassen. Auch dass viele - wenn nicht sogar alle - Verben sowohl einen zeitlichen als auch einen inhaltlichen Verlauf beschreiben und somit gleichzeitig zu zwei verschiedenen Aktionsklassen gezählt werden müssten, ist problematisch. Konsequent betrachtet, drückt jedes Verb einen zeitlichen Verlauf aus - sei es Anfang, Ende, einmalig oder ohne Begrenzung des Ablaufs. Dies lässt sich beispielsweise bei Verben des Veranlassens erkennen, die häufig mit der Vollendung eines Vorgangs identisch sind. So drückt das Verb sichern neben der Veranlassung, dass etwas sicher wird, auch das Ende des Prozesses des Sichermachens aus. Auch Nicolay warnt vor einer Vermengung von Aktionsart und (mehr oder weniger) verwandten Phänomenen. Dementsprechend behandelt sie 
semantische Faktoren, die nicht die zeitliche Struktur betreffen wie Agentivität und Kausativität, auch nicht als Teil der Aktionsart eines Verbs (Nicolay 2007: 2). Aufgrund dieser Kritikpunkte an der Unterteilung der Aktionsklassen in zeitliche und inhaltliche beschränke ich mich in dieser Arbeit auf die zeitlichen Aktionsklassen.

\subsubsection{Aktionsarten nach Vendler (1967)}

Die in dieser Arbeit unterschiedenen Klassen von Aktionsarten richten sich nach der bis heute als grundlegend geltenden Klassifizierung von Vendler (1967). Vendler unterscheidet Aktionsarten in State, Activity, Accomplishment und Achievement (1967: 102-103). Unter States versteht Vendler Verben, die einen dauerhaften Zustand beschreiben wie know, believe, love/hate und have/possess. Activitys dagegen sind Verben, die eine andauernde, aber nicht auf ein Ziel gerichtete Handlung bezeichnen wie work, play (chess), smoke und write. Dabei bezeichnet Vendler, wie oben erwähnt, je nach Kontext und Verbbedeutung write (books), paint oder think einmal als States und einmal als Activitys. Ich betrachte in dieser Arbeit bei der Zuordnung eines Verbs zu einer Aktionsart nur die eigentliche Verbbedeutung, die sich aber teilweise nur kontextbedingt offenbart. Rauchen sehe ich also, egal ob es sich um ein habituelles Rauchen eines Rauchers oder um ein momentanes Rauchen einer Zigarette handelt, immer als Activity an. Rauchen als Indikator für das Vorhandensein eines Feuers ist dagegen ein Zustand und somit zu den States zu zählen. Zur Aktionsart Accomplishment rechnet Vendler auf ein Ende zulaufende Handlungen wie grow up, make [a chair] und build [a house]. Achievements schließlich sind Verben, die singuläre Ereignisse inklusive Anfang und Ende ausdrücken, wie lose/find und get married (Vendler 1967: 99-111).

Für die Zuordnung konkreter Verben zu einer der Klassen werden die drei binären Parameter +/-durativ, +/-dynamisch und +/-telisch herangezogen, die sich in ähnlicher Form bereits bei Vendler (1967: 99-121) finden und durch Comrie exakt festgelegt wurden (1981: 41-51). Durative Verben umfassen zumindest eine gewisse Zeitdauer. Hierzu zählen States wie wissen, Activitys wie essen oder Accomplishments wie bauen. Dynamisch meint, dass ein Energieaufwand aufgebracht wird oder die Veränderung einer Situation oder eines Partizipanten geschieht. Abgesehen von State sind dementsprechend alle Aktionsarten dynamisch. Die Telizität unterscheidet, ob Verben einen Grenzpunkt in ihrer Verbbedeutung enthalten (=telisch) oder nicht (=atelisch). Während Verben wie wissen oder essen nicht auf ein Ende gerichtet sind beziehungsweise dieses nicht in ihrer spezifischen Verbsemantik enthalten, beschreiben Verben wie finden oder 
aufessen singuläre Ereignisse beziehungsweise Prozesse, die ohne einen Grenzpunkt ihre eigentliche Bedeutung verlieren (Comrie 1981: 41-51).

In Anlehnung an viele Arbeiten, die sich auf das System von Vendler (1967) berufen, wie Comrie (1981), Ehrich/Vater (1989), Krause (2002), Henriksson (2006), Rödel (2007) und Fischer (2018), spreche ich mich in dieser Arbeit zudem für eine Erweiterung der vierteiligen Klassifizierung Vendlers (1967) um die Kategorie Semelfactive aus. Dabei ergibt sich diese Kategorie als weitere Untergliederung von Achievement in Achievement und Semelfactive. Der Grund für diese Ergänzung liegt in einer Unterspezifizierung der Aktionsartengruppe Achievement nach Vendler (1967). Wenn man diese mit den oben genannten binären Parametern beschreibt, umfasst sie sowohl telische (z. B. finden) als auch atelische (z. B. nießen) Verben und widerspricht somit dem binären Prinzip. Dass gerade die Frage nach der Telizität von Verben wichtig ist, stellt unter anderem Nicolay (2007) heraus. Dabei äußere sich die Dominanz dieser Dichotomie unter anderem darin, dass dieser häufig die anderen aktionalen Merkmale untergeordnet werden und Verben in erster Linie nach ihrem Wert für Telizität klassifiziert werden (Nicolay 2007: 28).

Dadurch entsteht ein aus fünf Klassen von Aktionsarten bestehendes System, das sich tabellarisch wie folgt darstellen lässt:

Tab. 2: Klassen von Aktionsarten und deren Merkmalskombinationen

\begin{tabular}{lllll}
\hline Klasse & \multicolumn{2}{l}{ Merkmalskombination } & Beispielverben \\
\hline Activity & [+ durativ] & [+ dynamisch] & [- telisch] & essen, spielen \\
Accomplishment & [+ durativ] & [+ dynamisch] & [+ telisch] & aufessen, kommen \\
Achievement & [- durativ] & [+ dynamisch] & [+ telisch] & finden, ankommen \\
Semelfactive & [- durativ] & [+ dynamisch] & {$[-$ telisch] } & nießen, klopfen \\
State & [+ durativ] & [- dynamisch] & [- telisch] & wissen, sitzen \\
\hline
\end{tabular}

Fassen wir noch einmal zusammen: Tempus, Aspekt und Aktionsart sind unterschiedliche sprachliche Mittel zur zeitlichen Verortung von Situationen. Während Tempus und Aspekt grammatische Kategorien sind, ist die Aktionsart eine lexikalisch-semantische. Tempus und Aspekt werden also formal am Verb realisiert. Die Aktionsart ist dagegen der lexikalischen Semantik des Verbs bereits inhärent.

Tempus hat im Gegensatz zu Aspekt und Aktionsart eine Verweisfunktion, d. h. Sprecher können mit Hilfe des Tempus von einer Zeitstufe auf eine andere (oder dieselbe) verweisen. Aspekt und Aktionsart drücken den inneren zeitlichen 
Verlauf einer Situation aus. Beim Aspekt unterscheidet der Sprecher subjektiv (d. h. bei identischem Verb), ob die dargestellte Situation als Ganzes (=perfektiver Aspekt/abgeschlossen) oder als sich im Verlauf befindend (=imperfektiver Aspekt/unabgeschlossen) betrachtet wird. Die Aktionsart legt die objektiv inhärente Verlaufsweise einer Verbalhandlung fest. Dabei wird unterschieden, ob Verbalhandlungen durativ (=andauernd), dynamisch (=Veränderung einer Situation/eines Partizipanten etc.) und telisch (=zielorientiert) sind. Während die Aktionsart die einzelne Situation separiert vom Kontext beschreibt, liefert der Aspekt eine Beschreibung des Gesamtkontextes der Situation. Dementsprechend gibt es eine Schnittstelle zwischen Aktionsart und Aspekt. In Äußerungen und Sätzen, die abgesehen von der Aktionsart keine weiteren Hinweise auf die Abgeschlossenheit oder Unabgeschlossenheit der Gesamtperspektive einer Situation haben, zeigt die Aktionsart die Gesamtperspektive an. 